\title{
Effects of Electroacupuncture at BL60 on Formalin-Induced Pain in Rats
}

\author{
Kyung-Ha Chang, ${ }^{1}$ Ran Won, ${ }^{2}$ Insop Shim, ${ }^{3}$ Hyejung Lee, ${ }^{3}$ and Bae Hwan Lee ${ }^{1}$ \\ ${ }^{1}$ Department of Physiology and Brain Korea 21 Project for Medical Science, Yonsei University College of Medicine, \\ Seoul 120-752, Republic of Korea \\ ${ }^{2}$ Division of Health Science, Department of Biomedical Laboratory Science, Dongseo University, Busan 617-716, Republic of Korea \\ ${ }^{3}$ Acupuncture and Meridian Science Research Center, Kyung Hee University, Seoul 130-701, Republic of Korea
}

Correspondence should be addressed to Bae Hwan Lee, bhlee@yuhs.ac

Received 1 June 2011; Revised 23 November 2011; Accepted 23 December 2011

Academic Editor: Fengxia Liang

Copyright (C) 2012 Kyung-Ha Chang et al. This is an open access article distributed under the Creative Commons Attribution License, which permits unrestricted use, distribution, and reproduction in any medium, provided the original work is properly cited.

\begin{abstract}
Acupuncture was used to treat symptoms of pain in the ancient orient. The present study was conducted to determine the effects of electroacupuncture (EA) at the BL60 acupoint on male Sprague-Dawley rats. Each rat received EA at BL60 acupoint before formalin injection. Behavioral responses were recorded using a video camera and c-Fos immunohistochemistry was performed thereafter. Treatment of EA at BL60 significantly inhibited flinching behavior and c-fos expression induced by formalin injection into the paw, compared to a control group. These results suggest that electroacupuncture at BL60 acupoint may be effective in relieving inflammatory pain.
\end{abstract}

\section{Introduction}

Acupuncture was used to treat symptoms of pain in the ancient orient. Currently, acupuncture has garnered increasing interest as a therapeutic method for treating pain. Recently, electroacupuncture, applying electrical stimulation, is being actively studied. Electroacupuncture was developed to resolve the problems of manual acupuncture, that is, the inconvenient twirling procedure and the difficulty of maintaining constant frequency [1].

The BL60 (Kunlun) acupoint has been shown to be involved in visual information processing [2]. Deadman et al. [3] reported that acupuncture stimulation at BL60 acupoint was effective in treating eye disorders as well as head disorders. Studies indicated that the BL60 acupoint has analgesic effects on chronic low back pain [4] as well as hind limb pain [5]. Li et al. [6] reported that bilateral EA treatment at both BL60 and ST36 acupoints was effective in alleviating inflammatory pain. However, there have been no reports that electroacupuncture at BL60 by itself has an analgesic effect on inflammatory pain.
Inflammatory pain is known to cause abnormality in the nervous system, thereby causing consistent pain [7]. Formalin has been widely used in experiments with animal models of inflammatory pain because of several strong points: (1) it induces behaviors that can be easily observable [7]; (2) the responses to the moderate and continuous pain can be measured $[8,9]$; (3) anesthesia is not necessary, so that the behaviors of freely moving animals can be observed $[8,9]$. The early phase of pain responses after formalin injection is due to the direct injury of tissues, reflecting nociceptive pain, while the late phase is due to peripheral inflammation and central sensitization [10].

One of the methods used to measure pain and analgesia in animal experiments is the immunohistochemical detection of the c-Fos protein encoded by $\mathrm{c}$-fos, an oncogene. The $\mathrm{c}$-fos gene is an immediate early gene and is rapidly expressed in neurons of the central nervous system when nociceptive stimuli are applied to a peripheral area; for this reason c-Fos is currently widely used as a marker of pain [11,12].

The present study was performed to determine if electroacupuncture at the BL60 acupoint would alleviate 
nociceptive pain in the early phase and inflammatory pain in the late phase of responses to formalin injection. In order to determine the effects of electroacupuncture on formalininduced pain, a behavioral test and c-Fos immunohistochemical study were conducted.

\section{Methods}

2.1. Animals. Male Sprague-Dawley rats weighing about $250 \sim 300 \mathrm{~g}$ were used in this study. The animals were housed in cages equipped with the barrier system for SPF (specific pathogen free) animals. The system automatically maintained proper temperature $\left(22 \pm 2^{\circ} \mathrm{C}\right)$, humidity $(50 \pm$ $10 \%)$ and lighting ( $12 \mathrm{hr}$ of light/dark). The beddings of the cages were regularly changed (twice a week). All animal experiments were approved by the Institutional Animal Care and Use Committee of Yonsei University Health System.

2.2. Electroacupuncture. A stainless steel needle (diameter $0.25 \mathrm{~mm}$; length $15 \mathrm{~mm}$; Dongbang Acupuncture Inc., Boryeong, Republic of Korea) was used for electroacupuncture [13]. The needle was connected to a wire for better direct application of electric stimuli. The accurate application of electrical stimulation was confirmed in a preliminary study with the experimental animals. The acupoint selected for acupuncture was the BL60 acupoint, which is located at the ankle joint level between the external malleolus and tendo calcaneus in the hind limb [5].

The experiment was designed to determine the efficacy of pretreatment of electroacupuncture (acupuncture treatment before formalin injection) applied to the BL60 acupoint ipsilateral to formalin injection. For this purpose, the animals were divided into three groups: Group 1 was injected with formalin only (Formalin), Group 2 was treated with electrical stimulation by electroacupuncture before formalin injection (EA-For), and Group 3 was treated with needle insertion at the acupoint before formalin injection, but was not treated with real electrical stimulation (Sham-For).

All the animals received inhalation anesthesia of $2 \%$ enflurane $\left(95 \% \mathrm{O}_{2} / 5 \% \mathrm{CO}_{2}\right)$ prior to electroacupuncture and/or formalin injection. For the EA-For group, which was to receive electroacupuncture at the BL60 acupoint, electrical stimulation was applied using an electrical stimulator (A385, World Precision Instruments, Sarasota, FL, USA) and the Pulsemaster (A300, World Precision Instruments, Sarasota, FL, USA) at $1 \mathrm{~Hz}$ (2 ms pulses, $3 \mathrm{~mA}$ ) for $10 \mathrm{~min} \mathrm{[14,}$ 15]. The depth of insertion was $2-3 \mathrm{~mm}$. The Sham-For group was kept for 10 minutes with the needle inserted at the BL60 acupoint but no electrical stimulation was applied. The Formalin group was kept under the inhalation anesthesia without any treatment before formalin injection. After electroacupuncture, the animals were kept awake for $10 \mathrm{~min}$ in order to acclimate to the test environment.

2.3. Behavioral Test. Formalin is an aqueous solution of formaldehyde (37\%). A dilution of formalin in saline was used for the experiments herein: $50 \mu \mathrm{L}$ of $5 \%$ formalin was used for injection, which is widely used to induce maximum pain response $[16,17]$, while avoiding adverse phenomena, such as ceiling effect, backward walking, and freezing [16, 17].

After electroacupuncture, the experimental animals received formalin injection beneath the left plantar skin using a 29 gauge insulin syringe. Then, the animals were immediately put in an observatory chamber $(46 \times 26 \times$ $20 \mathrm{~cm}$ ) and video-recorded for $60 \mathrm{~min}$. The pain behaviors of the rats were analyzed by counting the flinching frequency of formalin-injected paws (number of flinches as 5 min passes) throughout the recording time. After videorecording, the rats were immediately subjected to c-Fos immunostaining.

2.4. c-Fos Immunohistochemistry. Normal rats as well as the three groups of rats above were used for c-Fos immunohistochemical study in order to observe changes in c-Fos immunoreactivity by comparing the experimental groups with normal rats. Under anesthesia with 25\% urethane $(1.25 \mathrm{~g} / \mathrm{kg}$, i.p.), the experimental animals were perfused through with $0.9 \%$ saline and $4 \%$ paraformaldehyde (in $0.1 \mathrm{M}$ phosphate buffer, $\mathrm{pH} 7.2$ ), and the L5 spinal cord section was removed. The removed tissues were fixed in $4 \%$ paraformaldehyde (in $0.1 \mathrm{M}$ phosphate buffer, $\mathrm{pH} 7.2$ ) for $4 \mathrm{hr}$ at $4^{\circ} \mathrm{C}$ and then kept in $30 \%$ sucrose solution overnight at $4^{\circ} \mathrm{C}$. The tissues were cut into $50 \mu \mathrm{m}$ thick slices using a Cryocut Microtome (Microm/HM500V, Walldorf, Germany). The total length of the L5 area was about $4000 \mu \mathrm{m}$, so that 80 slices of $50 \mu \mathrm{m}$ thickness were obtained, every 5 th slice of which was selected for study for a total of 16 slices. These 16 slices were subjected to free floating staining in the 24 -well plates containing $1 \mathrm{x}$ phosphate-buffered saline. The tissues reacted in a solution of $30 \%$ methanol with $1 \% \mathrm{H}_{2} \mathrm{O}_{2}$ for 30 minutes, followed by reactions in a solution with $3 \%$ normal goat serum (NGS), $1 \%$ bovine serum albumin (BSA), and $0.3 \%$ Triton$\mathrm{X}$ for $30 \mathrm{~min}$; and then, the c-Fos antibody (c-Fos antirat polyclonal IgG, 1500, Santa Cruz Biotechnology, Santa Cruz, CA, USA) was treated as the primary antibody and the mixture was kept overnight at $4^{\circ} \mathrm{C}$. Then, biotinylated antirabbit IgG ( $1: 200$, Vector, Burlingame, CA, USA) was treated as the secondary antibody and the mixture was allowed to react at room temperature for $2 \mathrm{hr}$, followed by treatment using the ABC kit (Vector, Burlingame, CA, USA) for $1 \mathrm{hr}$. 3,3-diaminobenzidine tetrahydrochloride (DAB, Sigma, St. Louis, MO, USA) was used for staining and the degree of staining was checked using a microscope (BX40 microscope, Olympus, Tokyo, Japan).

The number of the c-Fos positive neurons was obtained by calculating the mean number of neurons in 4 slices out of the 16 slices that went through the immunohistochemical procedure. A microscope (BX40, Olympus, Tokyo, Japan) was used at a magnification of $10 \mathrm{x}$ to check the lamina on the ipsilateral site to the formalin injection, in order to distinguish between lamina I-II and III-VI areas [18]. The MetaMorph software (ver. 4.6, Universal Imaging, Downingtown, PA, USA) was used to count the stained neurons, along with a microscope (BX51, Olympus) mounted with a CCD 
camera (Cool SNAP Photometrics, Roper Scientific, Tucson, $\mathrm{AZ}, \mathrm{USA}$ ) at a magnification of $10 \mathrm{x}$.

2.5. Statistical Analysis. The SPSS 15.0 program (SPSS Inc., Chicago, IL, USA) was used to compare the pain behaviors and the number of the c-Fos positive neurons in each experiment. Data were presented as mean \pm SEM, and statistical significance was given when $P$ values were less than 0.05 . As for the observation of behavioral responses, the flinching frequencies of each group, divided into the early phase and late phase responses, were analyzed by one-way ANOVA followed by Dunnett's multiple comparison test (2sided) for post hoc analysis. As for the immunostaining, the spinal cords were classified into lamina I-II and III-VI, and the numbers of $\mathrm{c}-$ fos positive neurons were compared by the same statistical method mentioned above.

\section{Results}

3.1. Behavioral Test. After formalin injection into the plantar paw, rats typically showed vivid flinching behaviors. These responses were characterized into the early phase (short rise and decay) and late phase (long-lasting for about $1 \mathrm{hr}$ ) responses. Thus, we classified the flinching behaviors into two phases and compared the effects of electroacupuncture on each phase of the formalin-induced pain behaviors.

The frequencies of the flinching behavior in the early phase were as follows: Group $137.04 \pm 16.76$ in the group injected only with formalin (Formalin; $n=12$ ); Group 2 $33.85 \pm 16.24$ in the group treated with electroacupuncture before formalin injection (EA-For; $n=17$ ); and Group $333.96 \pm 10.28$ in the group with needle insertion before formalin injection, but not treated with electrical stimulation (Sham-For; $n=13$ ). As shown in Figure 1, flinching behaviors did not show any significant difference in the early phase $\left(F_{2,31}=3.197, P>0.05\right)$.

The frequencies of the flinching behavior in the late phase were as follows: Group $1329.88 \pm 73.56$ in the group injected only with formalin (Formalin; $n=12$ ); Group 2 $243.44 \pm 109.33$ in the group treated with electroacupuncture before formalin injection (EA-For; $n=17$ ); and Group 3 $291.42 \pm 99.50$ in the group with needle insertion before formalin injection, but not treated with electrical stimulation (Sham-For; $n=13$ ). The EA-For group showed statistically significant decrease in pain response behaviors compared to the Formalin group $\left(F_{2,31}=5.017, P<0.05\right.$; oneway ANOVA followed by Dunnett's multiple comparison) (Figure 1). These data showed that the pre-treatment with electroacupuncture at BL60 significantly inhibited flinching behavior, compared to the control group.

3.2. c-Fos Immunohistochemistry. The number of c-Fos positive neurons in the dorsal horn was counted separately for superficial layers (lamina I-II) and deep layers (III-VI). The representative photographs of the c-Fos positive neurons of the individual groups are shown in Figure 2. As shown in Figure 2, the EA-For group showed a remarkable decrease

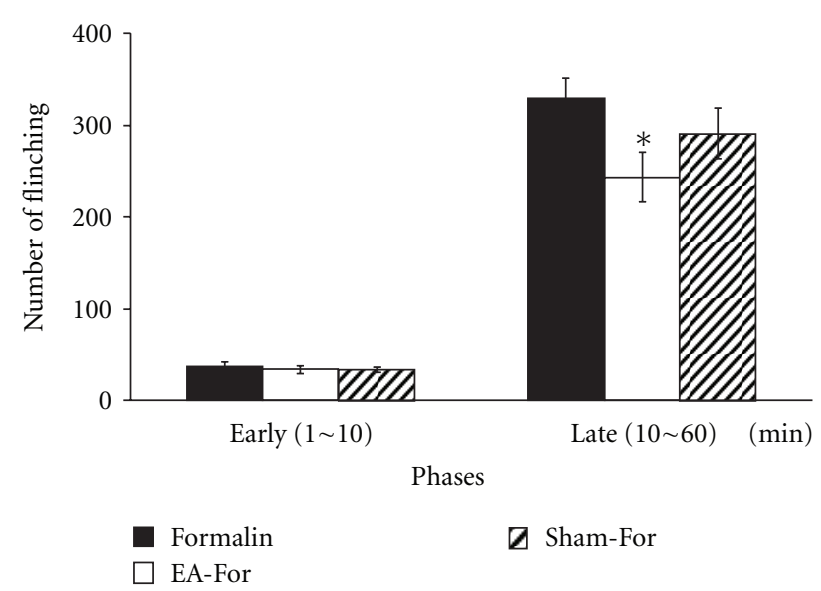

Figure 1: Effects of electroacupuncture on flinching numbers. Bar graphs were divided into early $(0-10 \mathrm{~min})$ and late phases (10-60 min). Experimental groups: Formalin formalin injection only group; EA-For electroacupuncture treatment at BL60 before formalin injection; Sham-For acupuncture needle insertion at BL60 but no electric stimulation before formalin injection. In the early phase, there were no significant differences between the groups. However, flinching numbers of the EA-For group were significantly decreased in the late phase. Each bar represents the group mean \pm $\operatorname{SEM}\left({ }^{*} P<0.05\right)$.

in the number of c-Fos positive neurons, compared to the Formalin group.

In order to compare the level of c-Fos immunoreactivity in the Formalin group Group 1 with pain-free animals, a normal naïve animal group Group 0 was added to this study. The numbers of c-Fos positive neurons in lamina I-II were as follows: Group 0, $250 \pm 30.68$ in the group with no treatment (Normal; $n=4$ ); Group 1, $2501 \pm 255.54$ in the group injected only with formalin (Formalin; $n=7$ ); Group 2, $1163.12 \pm 219.16$ in the group treated with electroacupuncture before formalin injection (EA-For; $n=8$ ); and Group 3, $1893 \pm 132.91$ in the group with needle insertion before formalin injection, but not treated with electrical stimulation (Sham-For; $n=10$ ) (left in Figure 3).

For lamina III-VI, the numbers were $268.75 \pm 45.38$ (Normal; Group 0), $2143.57 \pm 200.389$ (Formalin; Group 1), $985.62 \pm 195.79$ (EA-For; Group 2), and $1762 \pm$ 171.84 (Sham-For; Group 3), respectively (middle in Figure 3). The total numbers of the c-Fos positive neurons in lamina I through VI were $518.75 \pm 71.97,4645 \pm 372.30$, $2148.75 \pm 406.42$, and $3655 \pm 274.78$, respectively (right in Figure 3). When the Normal group Group 0 was compared to each group, the Formalin group Group 1 and the ShamFor group Group 3 showed a statistically significant increase in the number of the c-Fos-expressed neurons in each lamina (I-II: $F_{3,17}=23.316$; III-VI: $F_{3,17}=23.546$; I-VI: $F_{3,17}=33.101, P<0.05$; one-way ANOVA followed by Dunnett's multiple comparison). When the Formalin group Group 1, the EA-For group Group 2, and the Sham-For group Group 3 were compared, however, the EA-For group Group 2 showed a statistically significant decrease in the number of c-Fos positive neurons compared to the Formalin 


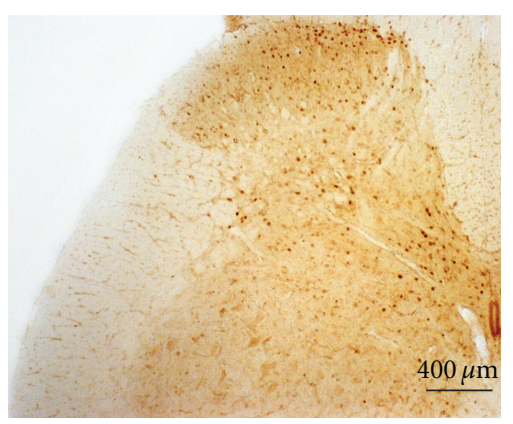

Formalin

(a)

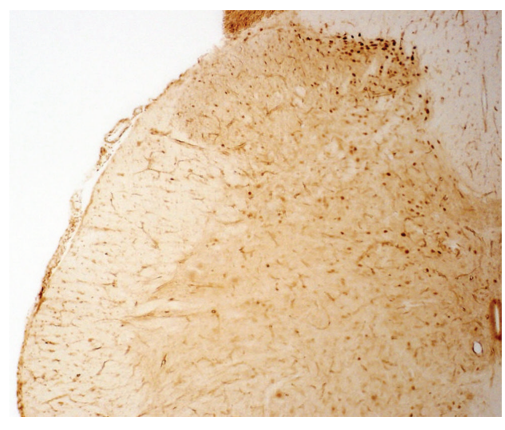

Sham-For

(c)

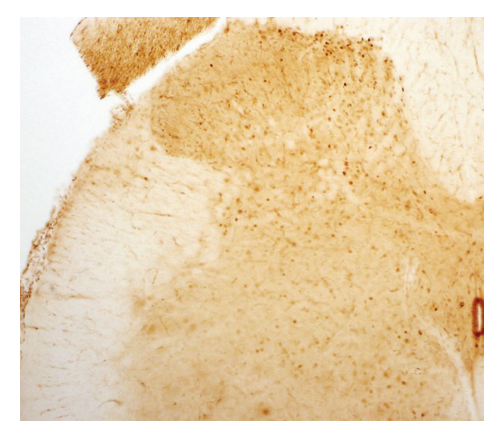

EA-For

(b)

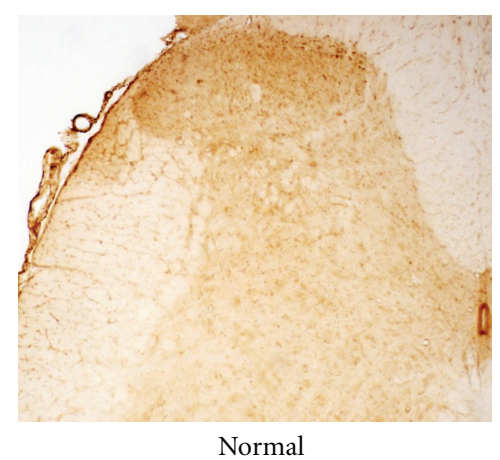

(d)

FIGURE 2: Representative photographs of c-Fos positive neurons in the spinal cord dorsal horn after electroacupuncture. (a) Formalin: formalin injection only group. (b) EA-For, electroacupuncture treatment at BL60 before formalin injection. (c) Sham-For, acupuncture needle insertion at BL60 but no electrical stimulation before formalin injection. (d) Normal: no treatment group.

group Group 1 in each lamina (I-II: $F_{2,14}=14.711$; III-VI: $F_{2,14}=15.647$; I-VI: $F_{2,14}=21.340, P<0.05$; one-way ANOVA followed by Dunnett's multiple comparison). These results showed that the pretreatment of EA at BL60 significantly inhibited c-Fos expression induced by formalin injection into the paw, compared to control group.

\section{Discussion}

Acupuncture has been used in Western medicine as well as Oriental medicine as an alternative treatment for pain-related disorders. In particular, electroacupuncture was developed to overcome the disadvantages of manual acupuncture, that is, the inconvenient twirling procedure and the difficulty of maintaining constant frequency.

Of inflammatory pain models, a formalin model was used in the present study in order to compare distinct biphasic nociceptive behavioral responses at both early and late phases after formalin injection. Electroacupuncture was pretreated before formalin injection in order to compare the effects of EA stimulation on both phases of formalininduced pain but was not posttreated because the effects of electroacupuncture stimulation on both the early and late phases only would be unobservable if electroacupuncture was applied after formalin injection, even though posttreatment resembles a natural clinical condition.
In the present study, EA stimulation with low-frequency ( $1 \mathrm{~Hz}, 3 \mathrm{~mA}$ ) at BL60 acupoint inhibited flinching behaviors in the late phase, but not in the early phase after formalin injection in rats. In electroacupuncture treatment, it has been shown that low-frequency stimulation produces prolonged analgesic effects relatively late in electroacupuncture; however, high-frequency stimulation produces shortlasting analgesic effects immediately after initiation of electroacupuncture [1]. Similarly, Lao et al. [18] showed that high frequency electroacupuncture produces the most potent anti-hyperalgesia in the early stage of complete Freund's adjuvant- (CFA-)induced hyperalgesia, while low-frequency electroacupuncture produces a prolonged inhibitory effect to reduce hyperalgesia.

As used in the present study, electrical stimulation with the intensity of $3 \mathrm{~mA}$ is known to be the maximum that conscious animals can withstand [18]. It was observed that the muscles around at the tip of the experimental animals' feet were trembling when electrical stimulation was applied with the intensity of $3 \mathrm{~mA}$. It was reported that this intensity of electroacupuncture stimulation has a therapeutic effect in inflammatory pain models [18].

According to Chang et al. [19], analgesic effects were observed at both early and late phases when electroacupuncture $(2,10,100 \mathrm{~Hz}$ frequency, $3 \mathrm{~mA}$ intensity, for $5 \mathrm{~min}$ ) was applied bilaterally at the ST36 (Zusanli) acupoint in mice. Kim et al. [17] reported that bilateral electroacupuncture 


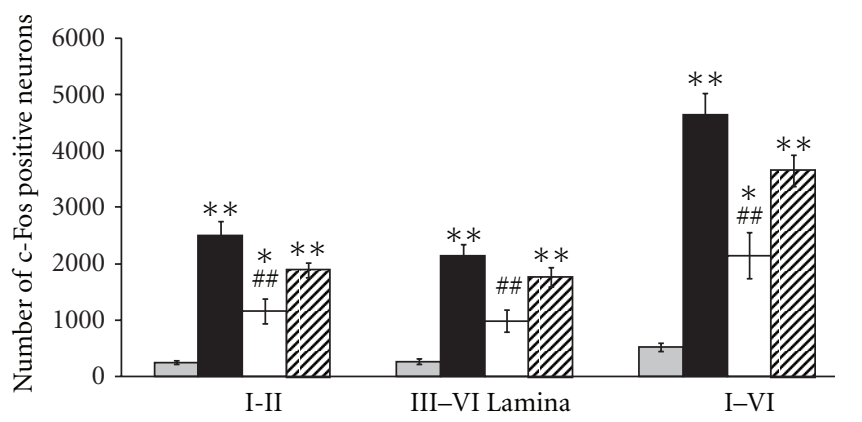

$\square$ Normal - Formalin

․ EA-For

Sham-For

III-VI

Figure 3: The number of c-Fos positive neurons in different groups. C-Fos positive cells were counted by MetaMorph software in lamina I-II and lamina III-VI at the L5 segment of the spinal cord ipsilateral to the site of formalin injection. Experimental groups were classified as follows: Normal: no treatment group; Formalin: formalin injection only group; EA-For, electroacupuncture treatment at BL60 before formalin injection; Sham-For, acupuncture needle insertion at BL60 but no electric stimulation before formalin injection. In each region, there was a significant decrease of c-Fos positive neurons when electroacupuncture was treated at BL60 compared to the Formalin group. On the other hand, in a comparison between Normal group and the other groups, there were notable increases in c-Fos positive neurons in the EA-For group and Sham-For group. Each bar represents the group mean \pm SEM $\left({ }^{*} P<0.05\right.$, ${ }^{* *} P<0.01$ for comparison between Normal group and all the other groups, one-way ANOVA followed by Dunnett's post hoc multiple comparison: \# $P<0.01$ for comparison between Formalin group and EA-For or Sham-For group, one-way ANOVA followed by Dunnett's post hoc multiple comparison).

( $2 \mathrm{~ms}, 10 \mathrm{~Hz}, 3 \mathrm{~mA}$, for $30 \mathrm{~min}$ ) at both HE7 (Shenmen) and PE7 (Daling) acupoints produces analgesic effects lasting nearly 1 hour in both early and late phases of formalininduced pain in rats. These results indicate that the painrelieving effects of electroacupuncture persist after EA stimulation cessation.

However, our behavioral results herein are not fully consistent with other studies $[17,19]$ that observed analgesic effects of EA in both early and late phases. The discrepancy may be attributable to differences in the acupoints treated, parameters in electroacupuncture stimulation, laterality, or experimental animals.

However, aspirin [20], nonsteroidal anti-inflammatory drugs (NSIADs) such as indomethacin and naproxen [21], compound 48/80, a histamine and serotonin depleter [9], and spinal anesthesia [22] can reduce late phase but not early phase formalin-induced pain. In relation to these studies, our study suggests that electroacupuncture stimulation at BL60 acupoint may be effective in relieving persistent inflammatory pain rather than in relieving acute pain produced by immediate activation of nociceptors in formalin test models.

There have been numerous studies that observed the pain-relieving effects of acupuncture [13, 16, 17, 23-25]. To our knowledge, however, there are no reports except for Kim et al. [26] and Zou et al. [27] to study the pain-relieving effects of acupuncture at the BL60 acupoint. Disappointingly, Kim et al. [26] did not find any analgesic effect with acupuncture at BL60. According to Zou et al. [27], patients with lumbar intervertebral disc herniation treated with EA combined with acupoint-injection at L4 Jiaji (EXB2), L5 Jiaji (EX-B2), Zhibian (BL54), Huantiao (GB30), Yanglingquan (GB34), Wizhong (BLA0), and Kunlun (BL60) showed significant improvement of pain, compared to a control group treated with electroacupuncture only. However, their study did not test the effects of electroacupuncture at BL60 acupoint itself. Therefore, our study may be the first to show the analgesic effects of electroacupuncture at BL60 acupoint in inflammatory pain.

Since Hunt et al. [28] reported c-Fos expression in response to peripheral nociceptive stimuli, c-Fos has been used as a neuronal marker of pain. Even though the role and significance of c-Fos expression in pain transmission is not fully understood [29], the remarkable correlation between the distribution of c-Fos positive neurons in the superficial layers (laminae I and II) of the lumbar spinal cord after formalin injection and the spinal distribution of pain-related afferent fibers innervating the plantar surface of the hind paw [30-32] suggests that the Fos protein may be expressed as a result of postsynaptic activation by painrelated afferents. In deep layers, few neurons receive direct pain-related afferents while most neurons receive convergent inputs from superficial layers [33], suggesting that Fos expression at the spinal cord reflects activation of second order neurons related to transmission of pain. Therefore, identification of c-Fos expression in the spinal dorsal horn, associated with paw flinching responses induced by formalin injection, can provide morphological anatomic evidence of pain and/or analgesia [34].

In relation to c-Fos expression in formalin-induced pain models, c-Fos seems to be expressed in superficial layers in relation to early phase pain and in deep layers in relation to late phase pain, while overall c-Fos expression appears to be concentrated in superficial lamina I-II [35]. However, in the present study, electroacupuncture treatment at BL60 acupoint reduced the number of $\mathrm{c}$-Fos positive neurons in not only superficial layer laminae I-II but also in deep layer laminae III-VI of the spinal cord at the L4-5 level, compared to a formalin injection only group. This result is consistent with other studies which observed a reduction of c-Fos expression in both superficial and deep layers of the spinal cord dorsal horn in the electroacupuncture-treated groups of a carrageenan-induced pain rat model $[16,36]$ and a formalin model [17]. Furthermore, it is considered that the mechanism of pain suppression by electroacupuncture at BL60 acupoint is due to its action at the spinal cord level, but not at the supra-spinal level. Also, the fact that there was a significant decrease in the number of c-Fos positive neurons in all parts of the lamina I-II and III-VI indicated that there is a close relationship between flinching responses and the number of c-Fos positive neurons during the treatment of electroacupuncture.

However, the mechanisms of analgesic effects of electroacupuncture treatment on formalin-induced pain are 
uncertain. Our results showed significant decrease in flinching response after the pretreatment of electroacupuncture. In our accompanying study, however, there was no significant reduction in licking response after the pretreatment of electric acupuncture (data are not shown). The flinching response is regarded to be a simple flexor reflex which is mediated at the spinal level, while the lifting or licking response is considered to be a more complex reflex which is mediated at the supraspinal level [36, 37]. It has been shown that the number of neurons expressing c-Fos increases when pain-transmitting neurons are activated in the spinal cord [28-33]. Our study showed that the number of cFos positive neurons decreased after electroacupuncture stimulation. This suggests that electroacupuncture relieves pain by inhibiting the transmission of pain at the level of the spinal cord.

In the spinal cord, the transmission of pain may be regulated in different ways. For example, activation of descending pain inhibition system from the brain may reduce the transmission of pain [38-40]. According to the gate control theory of Melzack and Wall [41], on the other hand, fastconducting somatosensory impulses by electroacupuncture stimulation arrive at the spinal cord dorsal horn to inhibit the activity of pain-transmitting neurons of the dorsal horn by blocking the input of pain information conducted from peripheral inputs [42-44]. Otherwise, electroacupuncture stimulation may induce diffuse noxious inhibitory controls (DNICs) in which analgesic effects by electroacupuncture treatment can be diffuse and unspecific, as the pain-relieving effect of electroacupuncture depends on the intensity of stimulation rather than the precise location thereof [4547]. Nevertheless, the detailed mechanisms of the analgesic effects of electroacupuncture treatment still remain to be determined. In order to elucidate these mechanisms, further studies will be needed.

\section{Conclusion}

The effects of electroacupuncture treatment at the BL60 acupoint (BL60) were examined in experimental animals with inflammatory pain induced by formalin. Judging from the fact that there was suppression of flinching behavior responses and of c-Fos expression upon the treatment of electroacupuncture therein, the treatment of electroacupuncture at BL60 acupoint is very effective in alleviation of inflammatory pain.

\section{Acknowledgment}

This paper was supported by the Basic Science Research Program through the National Research Foundation of Korea (NRF) funded by the Ministry of Education, Science and Technology (no. 2005-0049404).

\section{References}

[1] S. Andersson, Pain Control by Sensory Stimulation, Raven Press, NewYork, NY, USA, 1979.
[2] Z. H. Cho, S. C. Chung, J. P. Jones et al., "New findings of the correlation between acupoints and corresponding brain cortices using functional MRI," Proceedings of the National Academy of Sciences of the United States of America, vol. 95, no. 5, pp. 2670-2673, 1998.

[3] P. Deadman, M. Al-Khafaji, and K. Baker, "Some acupuncture points which treat headache," Journal of Chinese Medicine, vol. 56, pp. 5-18, 1998.

[4] J. G. Song, G. S. Lim, and K. S. Kim, "Effects of acupuncture on the plasma levels of $\beta$-endorphine, ACTH and cortisol in chronic low back pain," The Journal of Korean Acupuncture \& Moxibustion Society, vol. 6, pp. 63-82, 1989.

[5] N. Shankar, A. Varshney, A. Bhattacharya, and K. N. Sharma, "Electroacupuncture, morphine and clonidine: a comparative study of analgesic effects," Indian Journal of Physiology and Pharmacology, vol. 40, no. 3, pp. 225-230, 1996.

[6] W. M. Li, K. M. Cui, N. Li et al., "Analgesic effect of electroacupuncture on complete Freund's adjuvant-induced inflammatory pain in mice: a model of antipain treatment by acupuncture in mice," Japanese Journal of Physiology, vol. 55, no. 6, pp. 339-344, 2005.

[7] H. Wheeler-Aceto, F. Porreca, and A. Cowan, "The rat paw formalin test: comparison of noxious agents," Pain, vol. 40, no. 2, pp. 229-238, 1990.

[8] D. Dubuisson and S. G. Dennis, "The formalin test: a quantitative study of the analgesic effects of morphine, meperidine, and brain stem stimulation in rats and cats," Pain, vol. 4, no. 2, pp. 161-174, 1977.

[9] A. Tjolsen, O. G. Berge, S. Hunskaar, J. H. Rosland, and K. Hole, "The formalin test: an evaluation of the method," Pain, vol. 51, no. 1, pp. 5-17, 1992.

[10] M. Shibata, T. Ohkubo, H. Takahashi, and R. Inoki, "Modified formalin test: characteristic biphasic pain response," Pain, vol. 38, no. 3, pp. 347-352, 1989.

[11] E. Bullitt, "Expression of c-fos-like protein as a marker for neuronal activity following noxious stimulation in the rat," Journal of Comparative Neurology, vol. 296, no. 4, pp. 517-530, 1990.

[12] S. P. Hunt, A. Pini, and G. Evan, "Induction of c-fos-like protein in spinal cord neurons following sensory stimulation," Nature, vol. 328, no. 6131, pp. 632-634, 1987.

[13] S. Hao, O. Takahata, and H. Iwasaki, "Electroacupuncture potentiates the antinociceptive effect of intrathecal endomorphin-1 in the rat formalin test," Neuroscience Letters, vol. 287, no. 1, pp. 9-12, 2000.

[14] L. Lao, G. Zhang, F. Wei, B. M. Berman, and K. Ren, "Electroacupuncture attenuates behavioral hyperalgesia and selectively reduces spinal Fos protein expression in rats with persistent inflammation," Journal of Pain, vol. 2, no. 2, pp. 111-117, 2001.

[15] R. X. Zhang, L. Lao, L. Wang et al., "Involvement of opioid receptors in electroacupuncture-produced anti-hyperalgesia in rats with peripheral inflammation," Brain Research, vol. 1020, no. 1-2, pp. 12-17, 2004.

[16] Y. R. Wen, G. C. Yeh, B. C. Shyu et al., "A minimal stress model for the assessment of electroacupuncture analgesia in rats under halothane," European Journal of Pain, vol. 11, no. 7, pp. 733-742, 2007.

[17] J. H. Kim, Y. S. Gwak, I. Lee et al., "Antinociceptive effects of heterotopic electroacupuncture in formalin-induced pain," American Journal of Chinese Medicine, vol. 34, no. 4, pp. 565$574,2006$.

[18] L. Lao, R. X. Zhang, G. Zhang, X. Wang, B. M. Berman, and K. Ren, "A parametric study of electroacupuncture on 
persistent hyperalgesia and Fos protein expression in rats," Brain Research, vol. 1020, no. 1-2, pp. 18-29, 2004.

[19] F. C. Chang, H. Y. Tsai, M. C. Yu, P. L. Yi, and J. G. Lin, “The central serotonergic system mediates the analgesic effect of electroacupuncture on zusanli (ST36) acupoints," Journal of Biomedical Science, vol. 11, no. 2, pp. 179-185, 2004.

[20] P. Carrive and I. Meyer-Carrive, "Changes in formalinevoked spinal Fos expression and nociceptive behaviour after oral administration of Bufferin A (aspirin) and L-5409709 (ibuprofen + caffeine + paracetamol)," Pain, vol. 70, no. 2-3, pp. 253-266, 1997.

[21] S. Hunskaar and K. Hole, "The formalin test in mice: dissociation between inflammatory and non-inflammatory pain," Pain, vol. 30, no. 1, pp. 103-114, 1987.

[22] T. J. Coderre, A. L. Vaccarino, and R. Melzack, "Central nervous system plasticity in the tonic pain response to subcutaneous formalin injection," Brain Research, vol. 535, no. 1, pp. 155-158, 1990.

[23] M. T. Wu, J. C. Hsieh, J. Xiong et al., "Central nervous pathway for acupunture stimulation: localization of processing with functional MR imaging of the brain-preliminary experience," Radiology, vol. 212, no. 1, pp. 133-141, 1999.

[24] M. H. Cha, J. S. Choi, S. J. Bai et al., "Antiallodynic effects of acupuncture in neuropathic rats," Yonsei Medical Journal, vol. 47, no. 3, pp. 359-366, 2006.

[25] H. W. Kim, Y. B. Kwon, T. W. Ham et al., "Acupoint stimulation using bee venom attenuates formalin-induced pain behavior and spinal cord Fos expression in rats," Journal of Veterinary Medical Science, vol. 65, no. 3, pp. 349-355, 2003.

[26] H. Y. Kim, J. Wang, I. Lee, H. K. Kim, K. Chung, and J. M. Chung, "Electroacupuncture suppresses capsaicin-induced secondary hyperalgesia through an endogenous spinal opioid mechanism," Pain, vol. 145, no. 3, pp. 332-340, 2009.

[27] R. Zou, Y. Xu, and H. Zhang, "Evaluation on analgesic effect of electroacupuncture combined with acupoint-injection in treating lumbar intervertebral disc herniation," Zhongguo Gu Shang, vol. 22, pp. 759-761, 2009.

[28] S. P. Hunt, A. Pini, and G. Evan, "Induction of c-fos-like protein in spinal cord neurons following sensory stimulation," Nature, vol. 328, no. 6131, pp. 632-634, 1987.

[29] T. Herdegen and M. Zimmermann, "Immediate early genes (IEGs) encoding for inducible transcription factors (ITFs) and neuropeptides in the nervous system: functional network for long-term plasticity and pain," Progress in Brain Research, vol. 104, pp. 299-321, 1994.

[30] J. E. Swett and C. J. Woolf, "The somatotopic organization of primary afferent terminals in the superficial laminae of the dorsal horn of the rat spinal cord," Journal of Comparative Neurology, vol. 231, no. 1, pp. 66-77, 1985.

[31] Y. Sugiura, C. L. Lee, and E. R. Perl, "Central projections of identified, unmyelinated (C) afferent fibers innervating mammalian skin," Science, vol. 234, no. 4774, pp. 358-361, 1986.

[32] S. Maslany, D. P. Crockett, and M. D. Egger, "Organization of cutaneous primary afferent fibers projecting to the dorsal horn in the rat: WGA-HRP versus B-HRP," Brain Research, vol. 569, no. 1, pp. 123-135, 1992.

[33] C. J. Woolf and A. E. King, "Physiology and morphology of multireceptive neurons with C-afferent fiber inputs in the deep dorsal horn of the rat lumbar spinal cord," Journal of Neurophysiology, vol. 58, no. 3, pp. 460-479, 1987.

[34] J. Feng, F. Q. Huo, N. Jia et al., "Activation of muopioid receptors in thalamic nucleus submedius depresses bee venom-evoked spinal c-Fos expression and flinching behavior," Neuroscience, vol. 161, no. 2, pp. 554-560, 2009.

[35] C. Abbadie, B. K. Talyor, M. A. Peterson, and A. I. Basbaum, "Differential antribution of the two phases of the formalin test ot the pattern of cFos expression in the rat spinal cord: studies with remifntanil and lidocaine," Pain, vol. 69, pp. 101-110, 1997.

[36] Y. Q. Zhang, G. C. Ji, G. C. Wu, and Z. Q. Zhao, "Excitatory amino acid receptor antagonists and electroacupuncture synergetically inhibit carrageenan-induced behavioral hyperalgesia and spinal fos expression in rats," Pain, vol. 99, no. 3, pp. 525-535, 2002.

[37] K. Okuda, C. Sakurada, M. Takahashi, T. Yamada, and T. Sakurada, "Characterization of nociceptive responses and spinal releases of nitric oxide metabolites and glutamate evoked by different concentrations of formalin in rats," Pain, vol. 92, no. 1-2, pp. 107-115, 2001.

[38] C. Watanabe, K. Okuda, C. Sakurada, R. Ando, T. Sakurada, and S. Sakurada, "Evidence that nitric oxide-glutamate cascade modulates spinal antinociceptive effect of morphine: a behavioural and microdialysis study in rats," Brain Research, vol. 990, no. 1-2, pp. 77-86, 2003.

[39] B. T. Choi, J. Kang, and U. B. Jo, "Effects of electroacupuncture with different frequencies on spinal ionotropic glutamate receptor expression in complete Freund's adjuvant-injected rat," Acta Histochemica, vol. 107, no. 1, pp. 67-76, 2005.

[40] H. F. Guo, J. Tian, X. Wang, Y. Fang, Y. Hou, and J. Han, "Brain substrates activated by electroacupuncture of different frequencies (I): comparative study on the expression of oncogene c-fos and genes coding for three opioid peptides," Molecular Brain Research, vol. 43, no. 1-2, pp. 157-166, 1996.

[41] R. Melzack and P. D. Wall, "Pain mechanisms: a new theory," Science, vol. 150, no. 3699, pp. 971-979, 1965.

[42] J. S. Han, "Acupuncture: neuropeptide release produced by electrical stimulation of different frequencies," Trends in Neurosciences, vol. 26, no. 1, pp. 17-22, 2003.

[43] P. D. Wall and W. H. Swert, "Temporary abolition of pain in man," Science, vol. 155, no. 3758, pp. 108-109, 1967.

[44] K. Toda, "Peripheral nerve stimulation for producing the suppressive effect on the tooth pulp-evoked jaw opening reflex in rat: relation between stimulus intensity and degree of suppression," Experimental Neurology, vol. 76, no. 2, pp. 309317, 1982.

[45] K. Kawakita and M. Funakoshi, "Suppression of the jawopening reflex by conditioning A-delta fiber stimulation and electroacupuncture in the rat," Experimental Neurology, vol. 78, no. 2, pp. 461-465, 1982.

[46] D. Le Bars, A. H. Dickenson, and J. M. Besson, "Diffuse noxious inhibitory controls (DNIC). I. Effects on dorsal horn convergent neurones in the rat," Pain, vol. 6, no. 3, pp. 283304, 1979.

[47] Z. Bing, L. Villanueva, and D. Le Bars, "Acupuncture and diffuse noxious inhibitory controls: naloxone-reversible depression of activities of trigeminal convergent neurons," Neuroscience, vol. 37, no. 3, pp. 809-818, 1990. 


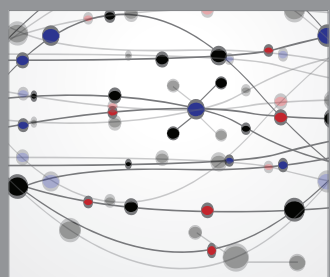

The Scientific World Journal
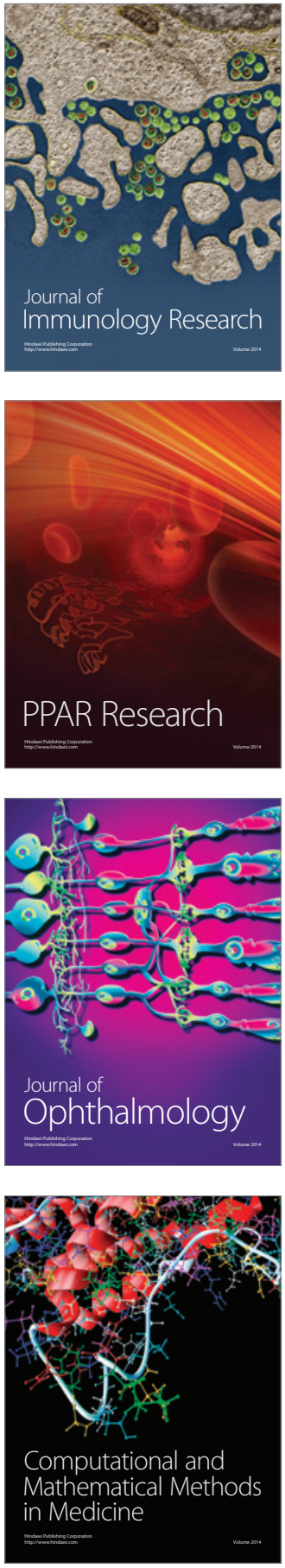

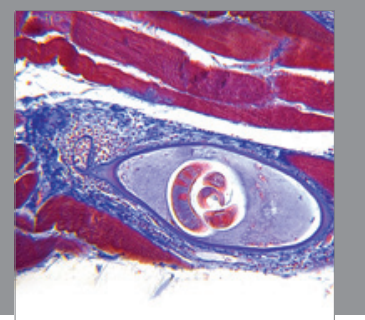

Gastroenterology

Research and Practice
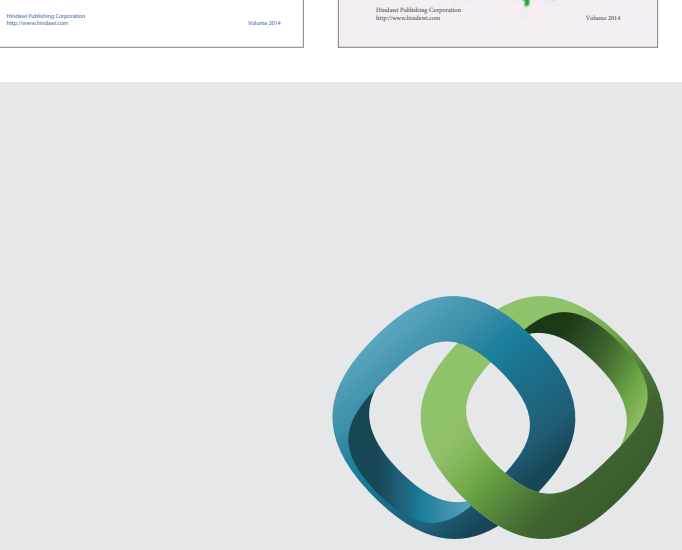

\section{Hindawi}

Submit your manuscripts at

http://www.hindawi.com
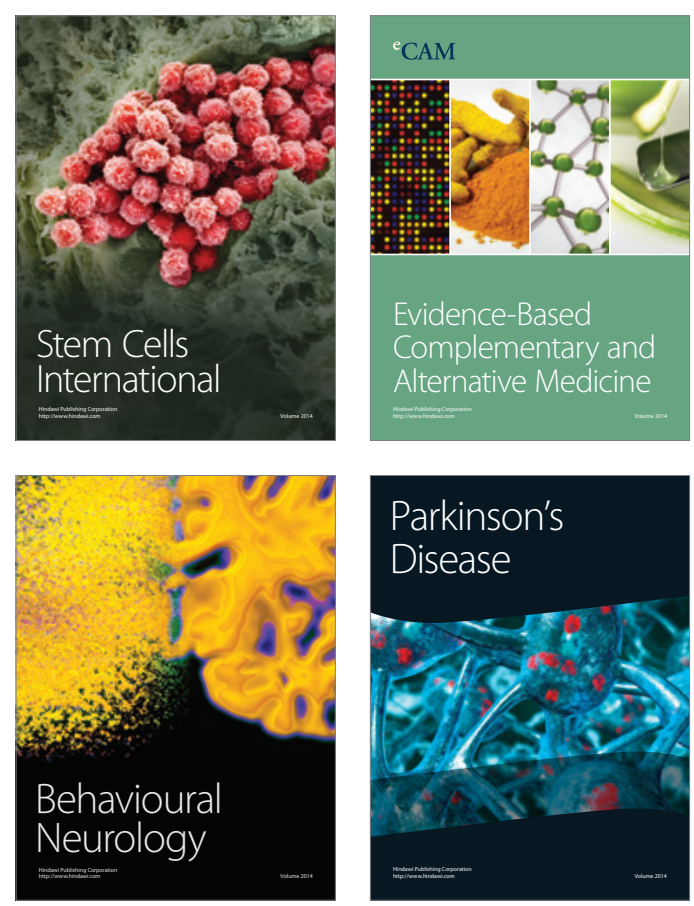

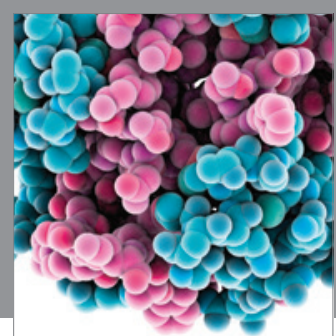

Journal of
Diabetes Research

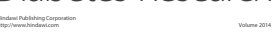

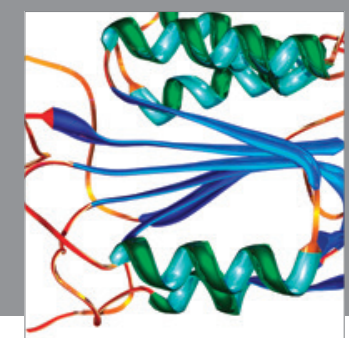

Disease Markers
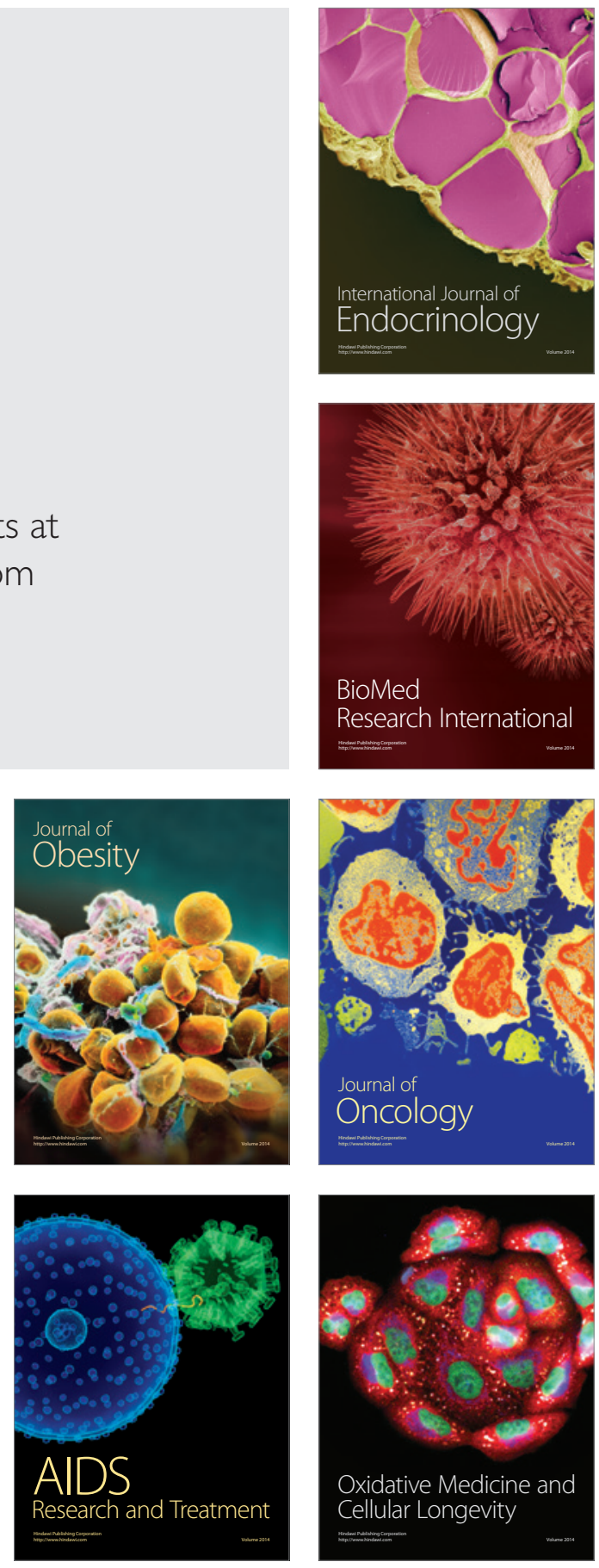